\title{
Critical role of two-dimensional island-mediated growth on the formation of semiconductor heterointerfaces
}

\author{
Esperanza Luna: A Álvaro Guzmán] and Achim Trampert \\ Paul-Drude-Institut für Festkörperelektronik, Hausvogteiplatz 5-7, 10117 Berlin, Germany \\ Gabriel Álvarez \\ Departamento de Física Teórica II, Facultad de Ciencias Físicas, Universidad Complutense, 28040 Madrid, Spain
}

(Dated: October 11, 2012)

\begin{abstract}
We experimentally demonstrate a sigmoidal variation of the composition profile across semiconductor heterointerfaces. The wide range of material systems (III-arsenides, III-antimonides, III-V quaternary compounds, III-nitrides) exhibiting such a profile suggests a universal behavior. We show that sigmoidal profiles emerge from a simple model of cooperative growth mediated by twodimensional island formation, wherein cooperative effects are described by a specific functional dependence of the sticking coefficient on the surface coverage. Experimental results confirm that, except in the very early stages, island growth prevails over nucleation as the mechanism governing the interface development and ultimately determines the sigmoidal shape of the chemical profile in these two-dimensional grown layers. In agreement with our experimental findings, the model also predicts a minimum value of the interfacial width, with the minimum attainable value depending on the chemical identity of the species.
\end{abstract}

PACS numbers: 68.35.Fx, 68.43.Fg, 68.55.A-, 81.05.Ea, 81.15.Hi

A central goal of modern materials physics is the control of interfaces down to the atomic level. In particular, the behavior of layered materials depends on the atomicscale structural roughness and chemical mixing across the interface [1]. Although abrupt interfaces between conventional semiconductors (such as III-V compounds) are fabricated and element profiles across these interfaces are obtained with atomic resolution, the relation between the layer growth processes and the parameters governing the interface formation and evolution is not satisfactorily understood. In this respect, there is an ongoing discussion about how interfaces can be quantitatively described on the basis of a growth model and whether there is a minimum interface width.

Recently Hulko et al. 2, 3] and Luna et al. [4 6] have shown empirically that experimental concentration profiles in III-V two-dimensional (2D) heterostructures, e.g. quantum wells $(\mathrm{QW})$ grown by molecular beam epitaxy (MBE), can be accurately reproduced by a sigmoidal function of the form $x(z)=x_{0} /[1+\exp (-z / L)]$. Here, $x_{0}$ denotes the nominal mole fraction of one of the species, $z$ is the position across the interface along the growth direction, and $L$ is the parameter quantifying the interface width ( $L$ is proportional to the widely reported length $W$, over which the concentration changes from $10 \%$ to $90 \%$ of its plateau value). Moreover, the accuracy of the sigmoidal fitting seems to be independent of the experimental technique used to obtain the element distribution [2, 5, 7] and, more interestingly, of the compound semiconductor. In this letter, we show that a sigmoidal profile emerges from a simple model of cooperative growth with $2 \mathrm{D}$ island formation. Furthermore, the use of a generalized sigmoidal expression gives a reliable and systematic quantification of the chemical interface. It sheds light on basic aspects of the early stages of heteroepitaxial growth, and permits to find a correlation between the profile and the interface properties in morphologically perfect epitaxial layers [8], which have been grown in the thermodynamically controlled Frank-vander-Merwe (FM) mode [9], not necessarily by MBE.

Experiments show that $L$ depends strongly on the combination of materials on both sides of the interface, but that heterostructures formed by the same material combination feature the same value of $L$ independently of their optimized growth methods [10] or substrate temperatures $T_{\mathrm{s}}$ 11]. It could be argued that different $T_{\mathrm{s}}$ might modify diffusion and intermixing processes at the interface and therefore account for the different values of $L$. This argument, however, does not explain the different values of $L$ for dissimilar heterostructures despite their same $T_{\mathrm{s}}$ or, conversely, that the same heterostructure features identical $L$ when grown at different $T_{\mathrm{s}}$. Therefore, the material interface property relation seems to be more complex than a mere increase in intermixing with increasing $T_{\mathrm{s}}$. Furthermore, the results suggest that there is a material-specific limitation in the interface width. The wide range of materials exhibiting a sigmoidal profile suggests a universal behavior determined by fundamental processes occurring during growth.

A sigmoidal growth has been reported to occur in biological, geological and chemical processes with cooperative effects [12], wherein the binding of one atom or molecule affects the binding of the subsequent atoms or molecules. The experimental observation of sigmoidal profiles at semiconductor heterointerfaces suggests that similar phenomena occur in materials science. We infer 


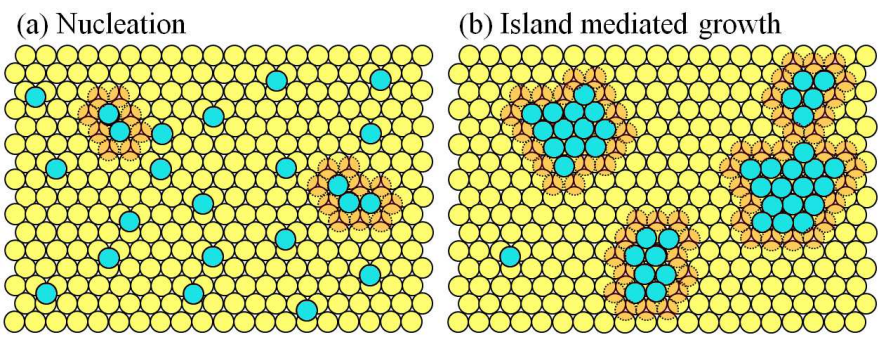

$\bigcirc$ bottom surface $\bigcirc$ occupied (top surface) $\bigcirc$ perimeter sites (top surface)

FIG. 1. (Color online) Schematic location of the chemisorbed atoms on a surface when (a) nucleation and (b) island growth dominates. The island-mediated growth proceeds via Eden cluster formation: in each growth step, empty perimeter sites next-neighbored to a seed (or to an occupied site) become occupied.

that the species (atoms or molecules, hereafter we will use both terms indistinctly) which are involved in the growth and interface formation process form a strong cooperative system, where the binding affinity (quantified by the sticking coefficient $s$ ) changes with the amount of atoms that have already been bound. More concretely, we assume that the rate of adsorption of A atoms (let us consider the growth of a pseudobinary semiconductor alloy $\mathrm{A}_{x_{0}} \mathrm{~B}_{1-x_{0}} \mathrm{C}$ on top of a binary compound $\mathrm{BC}$ ) is proportional both to the surface concentration of chemisorbed A atoms $\sigma$ and to the surface concentration of free A sites $\left(\sigma_{0}-\sigma\right)$, where $\sigma_{0}$ is the total surface concentration of A sites:

$$
\frac{d \sigma}{d t}=\frac{s_{1} J}{\sigma_{0}} \sigma(t)\left(\sigma_{0}-\sigma(t)\right)
$$

Here $s_{1}$ is a parameter that depends on the material system (we will see later that it corresponds to the initial island growth sticking coefficient) and $J$ is the impingement rate on an area of the substrate corresponding to one adsorption site [13, 14]. If we assume that, after fast local rearrangements not described by a simple kinetic equation, the thickness $z$ is proportional to the time variable $t$, then the variation of the surface coverage of $\mathrm{A}$ atoms $\theta=\sigma / \sigma_{0}$ is described by the differential equation:

$$
\frac{d \theta}{d z}=\frac{s_{1} J}{r} \theta(z)(1-\theta(z))
$$

where the constant $r$ is the mean growth rate of the pseudobinary alloy. The solutions of Eq. (2) with initial conditions between zero and one are sigmoidal functions. Equations (11) and (2) feature a surface-coverage dependent sticking coefficient $s(\theta)=s_{1} \theta(1-\theta)$. Incidentally, we mention that the experimentally measured value of the mole fraction $x$ is simply proportional to $\theta$.

Comprehensive investigations on the MBE growth of the II-VI compound CdTe showed that the sticking coefficients for both the $\mathrm{Cd}$ and the Te atoms were not constant [15]. Based on a remarkably good fitting of their data, Litz et al. suggested the existence of a precursor state during MBE growth, in which atoms are physisorbed to the growing surface in the manner of Kisliuk's theory of precursor-mediated adsorption [15]. In his theory, Kisliuk distinguished two types of physisorbed molecules, intrinsic precursor molecules (ipm) and extrinsic precursor molecules (epm), which are physisorbed above vacant and occupied chemisorption sites, respectively, albeit he assumed that adsorbed molecules are uncorrelated [16]. Ensuing computer simulations revealed that molecules adsorbed via an epm mechanism tend to aggregate into $2 \mathrm{D}$ clusters or islands [17]. Becker et al. [13, 14] extended Kisliuk's model to include these lateral positional correlations. In their model, Becker et al. addressed chemisorption as a combination of two mechanisms, each corresponding to the two types of precursor states introduced by Kisliuk: the ipm are responsible for a seeding process in which a molecule is directly chemisorbed above a vacant site and serves as a nucleus for the formation of an island, while epm are responsible for island growth by initial physisorption above an occupied chemisorption site followed by lateral jumps, until a vacant chemisorption site is reached. In this latter process, attractive adsorbate-adsorbate interactions enhance the precursor binding potential near the island edges, so that the epm will most likely become chemisorbed next to an already chemisorbed atom at the island edge, generating 2D compact Eden-type clusters [17 19], as it is illustrated in Fig. 1) Furthermore, Becker et al. proposed the additive contributions of the (ipm) nucleation and (epm) island growth terms to the sticking coefficient: for the nucleation process, they assumed a Langmuir form where the sticking coefficient is proportional to the fraction of empty sites, i.e., to $s_{0}(1-\theta)$ where $s_{0}$ is the initial sticking coefficient [9], while for the island growth term they assumed dependencies such as $\sqrt{1-\theta}$, which do not lead to pure sigmoidal profiles.

To gain insight into the phenomena underlying the interface formation, we have generalized Eq. (2) by adding the Langmuir dependence of the seeding process (which describes independent nucleation sites that adsorb no more than one adsorbate and leads to a random spatial distribution of nucleation centers on the surface) to our growth term (2). The latter describes the attractive adsorbate-adsorbate interaction previously mentioned through the $\theta(1-\theta)$ dependence. Thus, Eq. (2) is extended to:

$$
\frac{d \theta}{d z}=\frac{J}{r}\left[s_{0}(1-\theta(z))+s_{1} \theta(z)(1-\theta(z))\right],
$$

or, equivalently,

$$
\frac{d \theta}{d z}=\frac{1}{L\left(1+\theta_{0}\right)}(1-\theta(z))\left(\theta_{0}+\theta(z)\right) .
$$

Equations (3) and (4) feature the generalized sticking 


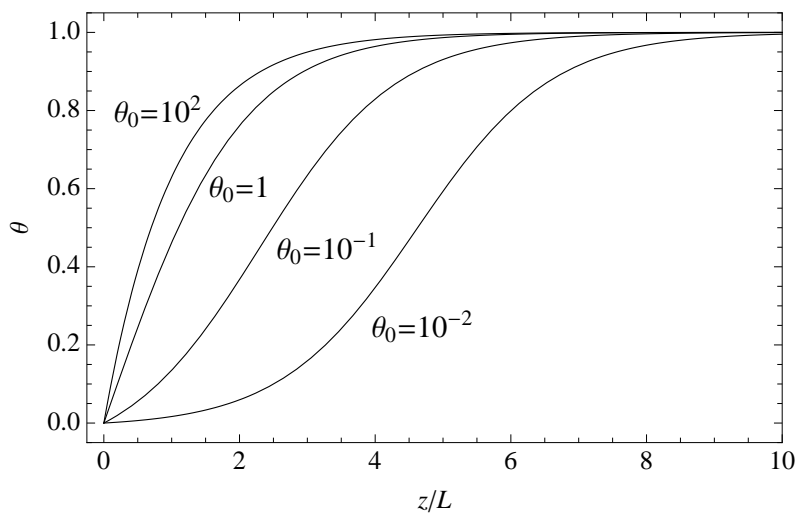

FIG. 2. Variation of the profiles given by Eq. (7) as $\theta_{0}=$ $s_{0} / s_{1}$ ranges from $10^{-2}$ to $10^{2}$. The limit $\theta_{0} \gg 1$ corresponds to nucleation, while the limit $\theta_{0} \ll 1$ corresponds to island growth.

coefficient

$$
s(\theta)=s_{0}(1-\theta)+s_{1} \theta(1-\theta),
$$

the fraction $\theta_{0}=s_{0} / s_{1}$ denotes the ratio of the initial sticking coefficients from the nucleation and island growth terms, respectively, and

$$
L=\frac{r / J}{s_{0}+s_{1}} .
$$

We remark that this equation gives the aimed connection between the interfacial profile, the growth process, and the material system. In particular, it gives the quantitative relation between the interface width $L$, the kinetic parameters $r$ and $J$, and the microscopic parameters $s_{0}$ and $s_{1}$. Furthermore, it refines the definition of an abrupt interface. Since $s_{0}+s_{1} \leq 2$, a non-vanishing interface width is predicted for any semiconductor heterointerface.

The general solution of Eq. (4) can be written in the form

$$
\theta(z)=1-\frac{1+\theta_{0}}{1+e^{\left(z-z_{0}\right) / L}}
$$

where $z_{0}$ is an integration constant that merely shifts the $z$ coordinate. Information on the main processes governing the growth and interface formation can be gained from inspection of Eq. (7). In Fig. 2 we plot the profiles obtained from Eq. (7) with $\theta(0)=0$ [which corresponds to $\left.z_{0}=-L \ln \left(\theta_{0}\right)\right]$ and $\theta_{0}$ ranging from $10^{-2}$ to $10^{2}$. The asymmetry of the profiles is a quantitative measure of the relative weight of the nucleation and the island growth terms: in the limit $\theta_{0} \gg 1$, i.e., $s_{0} \gg s_{1}$, where nucleation is the only mechanism, the profiles are very asymmetric and tend to $\theta(z)=1-\exp (-z / L)$; the asymmetry of the profiles decreases with $\theta_{0}$, and at $\theta_{0}=1$, i.e., when $s_{0}=s_{1}$, an inflection point appears in the profile; finally, in the limit $\theta_{0} \ll 1$ where island growth dominates, we recover the pure, symmetric sigmoidal profile $\theta(z)=1 /[1+\exp (-z / L)]$. Note, however, that if $s_{0}=0$ the solution of the differential equation (2) with $\theta(0)=0$ is identically zero, which shows that nucleation must always exist. However, in this case, 2D island growth occurs from the very early stages of the interface formation, since very few but already existing nuclei are sufficient to promote further atom attachment at their rims (Eden growth, cf. Fig. (1). Obviously this process is initially less efficient than seeding, and initially it takes more time to increase the composition profile to significative values.

We have analyzed experimental element profiles using the functional form in Eq. (7) and derived $\theta_{0}$ and $L$ for different III-V semiconductor heterostructures. We have investigated more than 60 samples comprising 2D morphologically perfect III-arsenides, III-antimonides, quaternary III-V alloys and III-nitrides heterostructures, which were fabricated using different methods. High-resolution and analytical transmission electron microscopy (TEM) techniques have been used to quantitatively determine the change in stoichiometry across the interfaces. Representative results are summarized in Table I. As observed, in all cases, regardless of the materials system and of the growth method, the largest values for $\theta_{0}$ are $\theta_{0} \approx 2 \times 10^{-2}$, which after due account of the accuracy, are consistent with a pure sigmoidal appearance of the experimental curves. Heterostructures formed by the same material combination feature the same $L$ value. The slight difference in $L$ for (In, Ga)As/GaAs interfaces formed by MBE and solid phase epitaxy (SPE), respectively, arises from experimental uncertainties at SPE. Despite the complexity in the growth and composition determination of (In, Ga) (As, N)/GaAs interfaces, high-quality heterostructures exhibit $L_{\mathrm{In}}=1.4-1.6 \mathrm{ML}$ and $L_{\mathrm{N}}=1-1.3 \mathrm{ML}$, even if grown at different laboratories. Additionally, $L$ varies with the combination of materials at both sides of the interface. As an example of these data and of the resulting fits, we show in Fig. 3(a) an experimental profile across an (Al, Ga)As/GaAs interface with $L=1.6 \mathrm{ML}$, while Figs. 3(b) and 3(c) correspond to the In and $\mathrm{N}$ distributions, respectively, across an (In, Ga)(As, N)/GaAs QW. In this case, the fit yields $L(\mathrm{In})=1.6 \mathrm{ML}$ and $L(\mathrm{~N})=1.3 \mathrm{ML}$ for the $(\mathrm{In}, \mathrm{Ga})(\mathrm{As}, \mathrm{N}) / \mathrm{GaAs}$ interface, while for the $\mathrm{GaAs} /(\mathrm{In}, \mathrm{Ga})(\mathrm{As}, \mathrm{N})$ interface we obtain $L(\mathrm{In})=1.7 \mathrm{ML}$ and $L(\mathrm{~N})=1.3 \mathrm{ML}$ (cf. Table 【). In all our experiments, we have not found neither significant asymmetric distribution with an exponential-like behavior nor curves reflecting $\theta_{0} \approx 1$. Furthermore, the experimental data are remarkably well reproduced by sigmoidal profiles, like those represented in Fig. 3, We thus conclude that, in spite of the ubiquitous nucleation term, the epm-mediated $2 \mathrm{D}$ island growth mechanism is the primary process at the forming interfaces and determines the general shape of the transition zone at the heterointerface. Note that, although the exact adsorption processes occurring in the epitaxy of semi- 
TABLE I. Parameters obtained from nonlinear fittings of the experimental profiles of 2D morphologically perfect III-V semiconductor heterostructures to the theoretical profile of Eq. (7). Growth methods: molecular beam epitaxy (MBE), solid phase epitaxy (SPE), metal-organic chemical vapor deposition (MOCVD). References are given for data taken from the bibliography. As a measure of the overall quality of the fit we include in the last column the coefficient of determination $R^{2}$.

\begin{tabular}{|c|c|c|c|c|c|c|}
\hline Material & Ref. & Growth method & $x_{0}(\%)$ & $\theta_{0}$ & $L(\mathrm{ML})$ & $R^{2}$ \\
\hline $\mathrm{Al}_{x_{0}} \mathrm{Ga}_{1-x_{0}} \mathrm{As} / \mathrm{GaAs}$ & & MBE & $29.46 \pm 0.02$ & $(1.7 \pm 0.8) \times 10^{-3}$ & $1.65 \pm 0.02$ & 0.9997 \\
\hline $\mathrm{In}_{x_{0}} \mathrm{Ga}_{1-x_{0}} \mathrm{As} / \mathrm{GaAs}$ & & MBE & $39.8 \pm 0.06$ & $(5.5 \pm 0.7) \times 10^{-3}$ & $1.31 \pm 0.01$ & 0.9996 \\
\hline $\mathrm{In}_{x_{0}} \mathrm{Ga}_{1-x_{0}} \mathrm{As} / \mathrm{GaAs}$ & & $\mathrm{SPE}$ & $34.2 \pm 0.02$ & $(2.3 \pm 1.8) \times 10^{-3}$ & $1.61 \pm 0.03$ & 0.9991 \\
\hline $\mathrm{In}_{x_{0}} \mathrm{Ga}_{1-x_{0}} \mathrm{As} / \mathrm{GaSb}$ & [20] & MBE & $49.2 \pm 2$ & & $1.0 \pm 0.1$ & \\
\hline $\mathrm{In}_{1-x_{0}} \mathrm{Ga}_{x_{0}} \mathrm{Sb} / \mathrm{InAs}$ & [21] & MBE & $81 \pm 3$ & $(1 \pm 1) \times 10^{-2}$ & $1.3 \pm 0.1$ & 0.9970 \\
\hline \multirow[t]{2}{*}{$(\mathrm{In}, \mathrm{Ga})(\mathrm{N}, \mathrm{As}) / \mathrm{GaAs}$} & & MBE & (In) $32.6 \pm 0.2$ & $(2.1 \pm 2.2) \times 10^{-3}$ & $1.62 \pm 0.03$ & 0.9990 \\
\hline & & & (N) $5.7 \pm 0.1$ & $(1.5 \pm 0.7) \times 10^{-2}$ & $1.3 \pm 0.1$ & 0.9892 \\
\hline \multirow[t]{2}{*}{$(\mathrm{In}, \mathrm{Ga})(\mathrm{N}, \mathrm{As}) / \mathrm{GaAs}$} & {$[4,22]$} & MBE & (In) $34.4 \pm 0.1$ & $(2.9 \pm 2.5) \times 10^{-3}$ & $1.45 \pm 0.03$ & 0.9996 \\
\hline & & & (N) $4.33 \pm 0.06$ & $(6.7 \pm 5.4) \times 10^{-3}$ & $1.0 \pm 0.1$ & 0.9988 \\
\hline $\mathrm{In}_{x_{0}} \mathrm{Ga}_{1-x_{0}} \mathrm{~N} / \mathrm{GaN}$ & & MOCVD & $23.9 \pm 0.2$ & $(1.8 \pm 0.4) \times 10^{-2}$ & $1.5 \pm 0.1$ & 0.9986 \\
\hline $\mathrm{In}_{x_{0}} \mathrm{Ga}_{1-x_{0}} \mathrm{~N} / \mathrm{GaN}$ & [23] & MOCVD & $7.15 \pm 0.02$ & $(1 \pm 1) \times 10^{-3}$ & $(0.177 \pm 0.002) \mathrm{nm}$ & 0.9995 \\
\hline
\end{tabular}
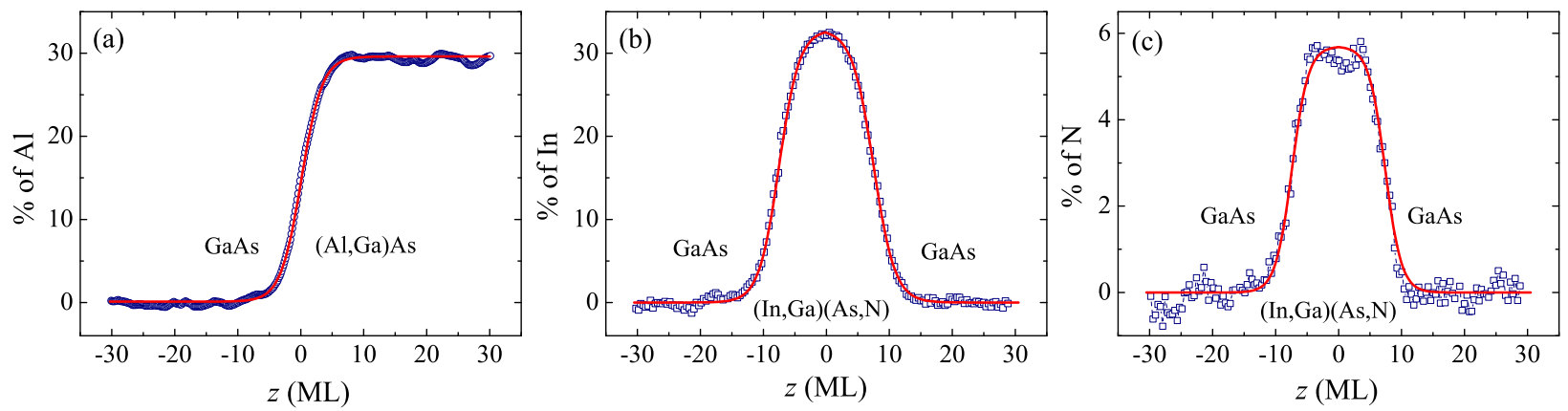

FIG. 3. (Color online) Experimental composition profile (open symbols) and sigmoidal function fits (solid line) across (a) an interface of $(\mathrm{Al}, \mathrm{Ga}) \mathrm{As} / \mathrm{GaAs}$. (b) and (c) represent the In and N profiles, respectively, across the interfaces in the QW structure $(\mathrm{In}, \mathrm{Ga})(\mathrm{As}, \mathrm{N}) / \mathrm{GaAs}$. The experimental relative error of the data points is typically $1.5 \%$ for $\mathrm{Al}$ and In and $4.6 \%$ for $\mathrm{N}$. (The growth direction goes from left to right).

conductors are generally unknown [24], it is very well established by reflection high-energy electron diffraction or scanning tunneling microscopy measurements that in the FM growth mode the completion of the layers occurs in an island-mediated $2 \mathrm{D}$ process $[25]$.

Furthermore, our comparative analysis of several III$\mathrm{V}$ heterostructures reveals a limitation in the interface width, which is specific to each material system, in agreement with the predicted limit in the value of $L$. As shown above, the interface width $L$ contains information both on the growth kinetics and on the adsorbate-surface relation (through $s_{0}$ and $s_{1}$ ). In general, the magnitude of the sticking coefficient and the adsorption dynamics are ultimately determined by the exact details of the potential energy surface (PES) that describes the particular molecular-surface interaction and is specific to a given molecule-surface system [9]. Our experimental findings suggest that, for samples grown under optimized conditions [10], the minimum attainable interface width is not kinetically driven, but thermodynam- ically driven through the adsorbate-surface interaction. The experimental results support this hypothesis. The width $L$ at the $\mathrm{A}_{x_{0}} \mathrm{~B}_{1-x_{0}} \mathrm{C} / \mathrm{BC}$ interface would be determined ultimately by the $\mathrm{A}_{\text {adsorbate }}-\mathrm{BC}_{\text {surface }} \mathrm{PES}$, which depends on the chemical identity of the surface and adsorbing atoms. Hence, the different values of $L$ for (Al, Ga)As/GaAs compared to (In, Ga)As/GaAs (cf. Table I) may arise from the different PES in the $\mathrm{Al}_{\text {adsorbate }}$ - GaAs surface and $\mathrm{In}_{\text {adsorbate }}-\mathrm{GaAs}_{\text {surface }}$ systems, respectively; and, similarly, the $\mathrm{In}_{\text {adsorbate }}$ $\mathrm{GaAs}_{\text {surface }}$ interaction will differ from the $\mathrm{In}_{\text {adsorbate }}$ $\mathrm{GaSb}_{\text {surface }}$ one, therefore leading to distinct values of $L$ in the (In, Ga)As/GaAs and (In, Ga)As/GaSb material systems, as experimentally observed. Finally, the intriguing identical $L$ for high-quality (In, Ga)(As, N)/GaAs QWs regardless of the growth procedure or laboratory is also explained because the PES would be similar in all cases. Equation (6) also predicts a dependence on the substrate orientation because the PES (and hence $s_{0}$ and $s_{1}$ ) depends on the crystallographic orientation of 
the surface above which growth proceeds.

Finally, we would like to point out that there is a close relation between Eq. (3) and a similar equation obtained in the context of silicon oxidation [26], where a sigmoidal behavior was also reported. Although seemingly dealing with a different problem, it has been recently shown that the initial stages of $\mathrm{Si}(001)$ oxidation are determined by a combination of a Langmuir-type adsorption and 2D island growth [26, 27] in close analogy with our study. Moreover, sigmoidal laws have been reported in the context of nanocrystals growth in solution [28], where recent investigations reveal a complex growth dynamics involving a $2 \mathrm{D}$ nucleation and growth process via atomic attachment from the solution phase [29, 30].

We conclude that a sigmoidal variation of the composition across an interface (or, in general, of any order parameter defining a system) directly arises from this specific growth dynamics characterized by strong cooperative interactions with $2 \mathrm{D}$ island formation and, therefore, it has a general character and is not restricted to MBE or MOCVD grown semiconductor heterointerfaces. We show that the prevalence of precursor-mediated $2 \mathrm{D}$ island growth over nucleation is the primary mechanism governing the interface development in $2 \mathrm{D}$ semiconductor heterostructures and responsible for the sigmoidal shape of the chemical profile at the interface. Moreover, while kinetics factors confirm as valuable control parameters for adjusting the interface width during the fabrication process, there is a minimum attainable interfacial width, which is dictated by the molecule-surface interaction potential and is exclusive for each material system considered, since the latter depends on the chemical identity of the species involved.

We would like to acknowledge C. Van de Walle (UCSB), K. H. Ploog and H. Riechert for fruitful discussions and S. Fölsch for a critical reading of the manuscript.

* luna@pdi-berlin.de

$\dagger$ Permanent address: ISOM and Dpto. Ingeniería Electrónica, ETSI Telecomunicación, Universidad Politécnica de Madrid, Spain.

[1] M. J. Hytch, M. G. Walls, and J.-P. Chevalier, Ultramicroscopy 83, 217 (2000).

[2] O. Hulko, D. A. Thompson, and J. G. Simmons, IEEE J. Sel. Top. Quant. Electron. 14, 1104 (2008).

[3] O. Hulko, D. A. Thompson, B. J. Robinson, and J. G. Simmons, J. Appl. Phys. 105, 073507 (2009).

[4] E. Luna, F. Ishikawa, P. D. Batista, and A. Trampert, Appl. Phys. Lett. 92, 141913 (2008).

[5] E. Luna, F. Ishikawa, B. Satpati, J. Rodriguez, E. Tournié, and A. Trampert, J. Crystal Growth 311, 1739 (2009).

[6] E. Luna, B. Satpati, J. B. Rodriguez, A. N. Baranov, E. Tournié, and A. Trampert, Appl. Phys. Lett. 96,
$021904(2010)$

[7] M. Müller, B. Gault, M. Field, G. J. Sullivan, G. D. W. Smith, and C. R. M. Grovenor, Appl. Phys. Lett. 100, 083109 (2012).

[8] The layers exhibit coherent interfaces and a structural interfacial roughness of $\pm 1 \mathrm{ML}$.

[9] K. W. Kolasinski, Surface Science: Foundations of Catalysis and Nanoscience, 2nd ed. (John Wiley \& Sons, Chichester, 2008).

[10] Optimized growth conditions refer to 2D grown heterostructures with smooth and coherent interfaces.

[11] For instance, $L=1.6 \mathrm{ML}$ for $\mathrm{Al}_{0.3} \mathrm{Ga}_{0.7} \mathrm{As} / \mathrm{GaAs}$ grown at $T_{\mathrm{s}}=580 \mathrm{C} ; L=1.3 \mathrm{ML}$ for $\mathrm{In}_{0.4} \mathrm{Ga}_{0.6} \mathrm{As} / \mathrm{GaAs}$ grown at $T_{\mathrm{s}}=420 \mathrm{C}$; whereas $L=1 \mathrm{ML}$ for $\operatorname{In}_{0.5} \mathrm{Ga}_{0.5} \mathrm{As} / \mathrm{GaSb}$ grown at $T_{\mathrm{s}}=420 \mathrm{C}$. Furthermore, in high-quality dilutenitride $(\mathrm{In}, \mathrm{Ga})(\mathrm{As}, \mathrm{N}) / \mathrm{GaAs}$ QWs, $L_{\mathrm{In}}=1.4-1.6 \mathrm{ML}$ and $L_{\mathrm{N}}=1-1.3 \mathrm{ML}$ regardless of $T_{\mathrm{s}}=375 \mathrm{C}$ or $420 \mathrm{C}$, even if grown at different laboratories.

[12] Y. Zhang, L. R. Manning, J. Falcone, O. Platt, and J. M. Manning, J. Biol. Chem. 278, 39565 (2003); H. W. Ducklow and S. M. Hill, Limnol. Oceanogr. 30, 239 (1985); F. Gugumus, Polym. Degrad. Stabil. 52, 159 (1996).

[13] O. M. Becker and A. Ben-Shaul, Phys. Rev. Lett. 61, 2859 (1988).

[14] O. M. Becker, J. Chem. Phys. 96, 5488 (1992).

[15] T. Litz, T. Behr, D. Hommel, A. Waag, and G. Landwehr, J. Appl. Phys. 72, 3492 (1992).

[16] P. Kisliuk, J. Phys. Chem. Solids 3, 95 (1957).

[17] E. S. Hood, B. H. Toby, and W. H. Weinberg, Phys. Rev. Lett. 55, 2437 (1985).

[18] J. W. Evans, Rev. Mod. Phys. 65, 1281 (1993).

[19] Eden growth was introduced in M. Eden, Proc. Fourth Berkeley Symp. on Math. Statist. and Prob. 4, 223 (1961) as a model for the formation of cell colonies in which each cluster grows following a simple geometrical rule: starting from an already existing single occupied lattice site or nucleation center, empty adjacent sites are occupied in each subsequent growth step; as a result of this mechanism Eden clusters have a compact structure.

[20] T. Taliercio, A. Gassenq, E. Luna, A. Trampert, and E. Tournié, Appl. Phys. Lett. 96, 062109 (2010).

[21] K. Mahalingam, K. G. Eyink, G. J. Brown, D. L. Dorsey, C. F. Kisielowski, and A. Thust, Appl. Phys. Lett. 88, 091904 (2006).

[22] J.-M. Chauveau, A. Trampert, K. H. Ploog, and E. Tournié, Appl. Phys. Lett. 84, 2503 (2004).

[23] T. J. Prosa, P. H. Clifton, H. Zhong, A. Tyagi, R. Shivaraman, S. P. DenBaars, S. Nakamura, and J. S. Speck, Appl. Phys. Lett. 98, 191903 (2011).

[24] P. Kratzer and M. Scheffler, Phys. Rev. Lett. 88, 036102 (2002).

[25] M. A. Herman, W. Richter, and H. Sitter, Epitaxy: Physical Principles and Technical Implementation (Springer Verlag, Berlin, 2004).

[26] M. Suemitsu, Y. Enta, Y. Miyanishi, and N. Miyamoto, Phys. Rev. Lett. 82, 2334 (1999).

[27] S. Ohno, H. Kobayashi, F. Mitobe, T. Suzuki, K. Shudo, and M. Tanaka, Phys. Rev. B 77, 085319 (2008).

[28] K. Biswas, N. Varghese, and C. N. R. Rao, Small 4, 649 (2008).

[29] H. Zheng, R. K. Smith, Y. Jun, C. Kisielowski, U. Dahmen, and A. P. Alivisatos, Science 324, 1309 (2009).

[30] B. Lim, H. Kobayashi, P. Camargo, L. Allard, J. Liu, and Y. Xia, Nano Res. 3, 180 (2010). 